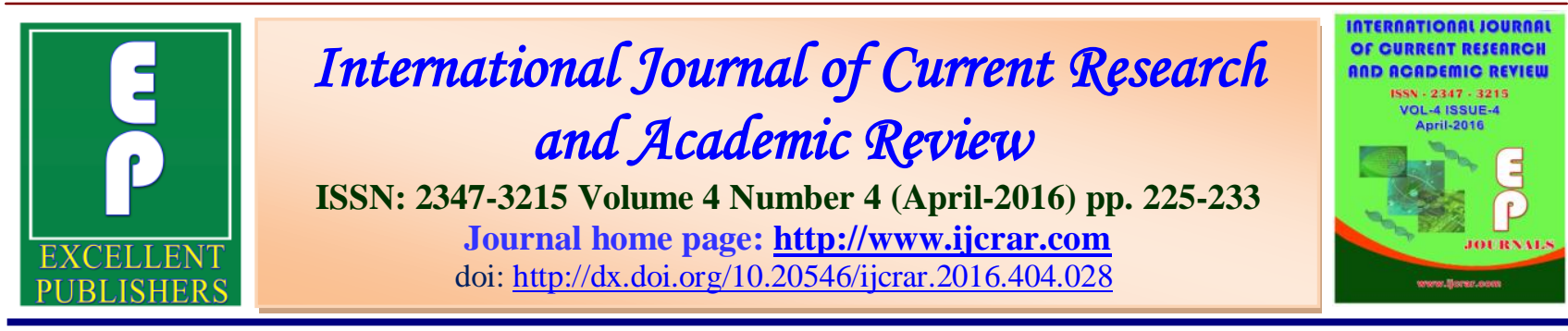

\title{
Effect of hydrochlorothiazide and spironolactone on reducing proteinuria among patients with diabetic nephropathy treated by angiotensin converting enzyme inhibitors
}

\author{
Hamid Noshad $^{1 *}$, Taher Manzary ${ }^{2}$ and Amir Teimouri-Dereshki ${ }^{3}$ \\ ${ }^{1}$ Professor of Nephrology, Chronic Kidney Disease Research Center, Faculty of Medicine, \\ Tabriz University of Medical Sciences, Tabriz, Iran \\ ${ }^{2}$ Resident of Internal Medicine, Imam Reza Hospital, Department of Internal Medicine, Faculty \\ of Medicine, Tabriz University of Medical Sciences, Tabriz, Iran \\ ${ }^{3}$ Students, Research Committee, Faculty of Medicine, Tabriz University of Medical Sciences, \\ Tabriz, Iran
}

*Corresponding author

\section{KEYWORDS}

Diabetic nephropathy, Proteinuria, Hydrochlorothiazide, Spironolactone

\section{A B S T R A C T}

Diabetic nephropathy is one of the leading causes of the end stage renal disease (ESRD) in all over the world. Overriding of renin-angiotensin-aldosterone system (RAAS) is considered as one of the important mechanisms of renal damage among diabetic patients. Treatment with RAAS inhibitor drugs, angiotensin converting enzyme inhibitors and angiotensin receptor blockers (ACEIs and ARBs) reduces the rate of renal involvement and consequently progression of diabetic nephropathy toward ESRD. The aim of this study was comparison of antiproteinuric effects of Hydrochlorothiazide (HCTZ) with Spironolactone in patients with diabetic nephropathy who were treated with ACEIs. In a randomized clinical trial in Sheikh-ol-raies university clinic, 90 hypertensive diabetic patients who were receiving Enalapril because of diabetic nephropathy underwent combination treatment with HCTZ and Spironolactone as two different groups. Urine albumin decreased from $1342 \pm 927 \mathrm{mg} / \mathrm{day}$ at the beginning of the study, to $1093 \pm 814 \mathrm{mg} /$ day among HCTZ group, and from $1183 \pm 916 \mathrm{mg} /$ day to $955 \pm 786 \mathrm{mg} /$ day in spironolactone group. but these differences was not statistically significant $(\mathrm{P}=0.9)$. Furthermore systolic blood pressure (SBP) decreased from $145 \pm 15$ to $135 \pm 16 \mathrm{mmHg}$ in HCTZ group and from $151 \pm 12$ to $145 \pm 16 \mathrm{mmHg}$ among Spironolactone group. In HCTZ group SBP decreased more significantly $(\mathrm{P}=0.022)$. Among Spironolactone receivers, serum potassium increased significantly. This difference was statistically significant $(\mathrm{P}=0.007)$. In our study, there were no statistically significant differences in decreasing albuminuria or reducing blood pressure between these two groups but, because HCTZ did not cause any hyperkalemia, combination of ACEIs with HCTZ is preferable. 


\section{Introduction}

Diabetes mellitus (DM) is considered as the most common endocrine disorder in the world and it is responsible for about 4 million deaths annually (1).

The prevalence of DM was estimated about 6.4 percent among adults (20-79 years old) in 2010 and was also estimated to increase up to 7.9 percent by 2030 (2). There are two major subtypes of DM, type one and type two. Type one DM is because of complete or partially depletion of insulin, in contrast; type two DM is a group of heterogeneous metabolic abnormalities that all of them lead to insulin resistance (1).

Furthermore type one DM is an autoimmune process that leads to destroying beta islet cells of pancreas and this usually occurs before third decade of age (3). Both types of DM can lead to multiple vascular and non vascular complications. diabetic retinopathy, diabetic nephropathy (DN), diabetic neuropathy, cardiovascular and cerebrovascular disease and peripheral artery disease are considered as the most important vascular complications. one of the most common of them that can also be preventable is diabetic nephropathy (4).

The prevalence of DN is estimated about $20 \%$ to $30 \%$ of all diabetic patients (type 1 and type 2). Hypertension and proteinuria are considered as risk factors of the progression of DN toward end stage renal disease (ESRD), and decreasing the range of proteinuria seems to be of utmost importance in slowing the rate of GFR decline $(5,6)$.

Different studies have raised the possibility that rennin-angiotensin-aldosteron system (RAAS) plays an important role in mediating renal damage (7). ACEIs and ARBs are both antihypertensive drug groups that are used as main treatment in hypertensive diabetic patients. In patients whom their blood pressure could not appropriately be controlled by each of above drug groups, another drug must be added to regimen (7).

Numerous studies have established the positive effect of HCTZ added to ACEIs and ARBs on reducing proteinuria among hypertensive patients without $\operatorname{DM}(8,9,10)$ and with DM (11).

Treatments with ACEIs and ARBs have showed binary effects on serum aldosterone in recent studies. Although they initially suppress its levels, but eventually in $40 \%$ of patients long term treatment may return plasma aldosterone to pretreatment levels. It is known as aldosterone escape phenomenon. So aldosterone antagonists such as spironolactone seems to be helpful when added to above treatments (12).

Recent studies showed that, the antiproteinuric effect of spironolactone is not completely related to its antihypertensive effect $(12,13,14)$. Some studies also show that may unknown antiproteinuric effects of HCTZ other than decreasing blood pressure in diabetic patients (11).

The positive effects of HCTZ and Spironolactone on reducing proteinuria among diabetic patients with diabetic nephropathy has been proven by recent studies, therefore we aimed to compare their antiproteinuric effects between diabetic patients who were treated with ACEIs because of diabetic nephropathy.

\section{Materials and Methods}

We compared the anti-proteinuric effects of spironolactone and HCTZ on reducing the 
proteinuria among hypertensive diabetic patients with diabetic nephropathy in a randomized clinical trial.

This study is approved by local ethical committee and Iranian randomized clinical trial center (IRCT). Ninety diabetic patients with proteinuria and hypertension, who were receiving ACEIs, referred to Sheikh-olRaeis university clinic and enrolled in our study.

Inclusion criteria were: Blood pressure more than $130 / 90 \mathrm{mmHg}$ in a patient who was receiving ACEIs, Proteinuria more than 300 mg/day Exclusion criteria were: Serum potassium more than $5 \mathrm{mEq} / \mathrm{dl}$, Glomerular filtration rate lower than $30 \mathrm{cc} / \mathrm{min}$, Secondary proteinuria not related to DM and hypertension (eg. Heart failure, urinary tract infections, etc.).

After explaining the patients about the safety of the drugs, possible risks of hyperkalemia and also ability of leaving the study voluntarily, they signed a written consent then enrolled in our study.By the way this study was ethically approval by Medical Ethics Committee of the Tabriz University of Medical Sciences.

Ninety diabetic patients who were receiving Enalapril $20 \mathrm{mg} /$ day because of their proteinuria and had the inclusion criteria were randomly chosen and then divided in two matched subgroups based on the age, sex, range of proteinuria and GFR.

One group underwent spironolactone treatment (25 mg/day) and other group treated with HCTZ (25 mg/day).

The range of proteinuria, blood pressure, serum urea, serum creatinine, serum sodium, serum potassium and serum albumin level were evaluated before study and then 3 months after beginning of study. Because of the risk of hyperkalemia, especially among spironolactone group, serum potassium and blood pressure were evaluated one week after beginning of the study and then monthly, in both groups. Every patient achieved a code and randomly received the drug by a third person. The laboratory was not aware of patient's drugs and study was double blinded.

Statistical analysis was performed with SPSS 16 by using repeated measurement of ANOVA test to analyze data. Mean values reported as mean \pm SD. $P$ values $<0.05$ were considered statistically significant.

\section{Results and Discussion}

Ninety patients were enrolled in our study: $48(59.3 \%)$ males and $33(40.7 \%)$ females. Nine patients who were treated with spironolactone, excluded because of high serum potassium level (more than $5 \mathrm{mEq}$ /L).

The mean age of patients was $44.4 \pm 4.6$ years old (youngest patient was 38 years old and oldest one 65 years old) (Table 1).

In the group of HCTZ, range of albuminuria was $1342 \pm 927 \mathrm{mg} /$ day at the beginning of the study and it was decreased to $1093 \pm 814$ $\mathrm{mg} /$ day at the end of the study ( $\mathrm{P}$ value < 0.0001). In Spironolactone group, range of albuminuria decreased from $1183 \pm 916$ $\mathrm{mg} /$ day at the beginning to $955 \pm 786 \mathrm{mg} /$ day at the end of study $(\mathrm{P}$ value $<0.01)$. The differences in albuminuria between these two groups were not statistically significant $(\mathrm{P}$ value $=0.33)$.

In HCTZ group, systolic blood pressure decreased form $145 \pm 15 \mathrm{mmHg}$ at the beginning to $135 \pm 16 \mathrm{mmHg}$ at the end of the study. 
In spironolactone group, systolic blood pressure decreased from $151 \pm 12 \mathrm{mmHg}$ at the beginning to $145 \pm 16 \mathrm{mmHg}$ at the end of the study. In both groups it was statistically significant $(\mathrm{P}<0.01)$. But HCTZ reduced systolic blood pressure more than spironolactone and it was statistically significant $(\mathrm{P}=0.02)$.

In HCTZ group serum potassium was decreased from $4.24 \pm 0.33 \mathrm{mEq} / \mathrm{L}$ to $4 \pm 0.19$ $\mathrm{mEq} / \mathrm{L}$ at the end of the study, in contrast serum potassium level was increased from $4.19 \pm 0.33 \mathrm{mEq} / \mathrm{L}$ to $4.31 \pm 0.32 \mathrm{mEq} / \mathrm{L}$ at the end of the study. This was statistically significant $(\mathrm{P}<0.01)$ (Fig. 1).

Diastolic blood pressure decreased from $86 \pm 8 \mathrm{mmHg}$ at the beginning of the study to $77 \pm 6 \mathrm{mmHg}$ at the end of the study. In HCTZ group and decreased from $87 \pm 8$ $\mathrm{mmHg}$ at the beginning to $78 \pm 7$ at the end of the study in Spironolactone groups. In both groups it was statistically significant $(\mathrm{P}<0.01)$ but these differences in diastolic blood pressure between two groups were not statistically significant either $\quad(\mathrm{P}=0.33)$ (Fig.2).

In the HCTZ group, serum albumin was increased from $3.43 \pm 0.38 \mathrm{mg} / \mathrm{dl}$ at the beginning to $3.55 \pm 0.35 \mathrm{mg} / \mathrm{dl}$ at the end of the study and serum albumin was also increased from $3.39 \pm 0.43 \mathrm{mg} / \mathrm{dl}$ to $3.53 \pm 0.35 \mathrm{mg} / \mathrm{dl}$ in Spironolactone group. In both groups it was statistically significant $(\mathrm{P}=0.02)$, but these differences were not statistically significant $(\mathrm{P}=0.79)$ (Fig.3).

In our study 9 patients ( all were receiving Spironolactone) were excluded because of high serum potassium level ( $\mathrm{k}>5.5 \mathrm{mEq}$ /L). In HCTZ group, serum creatinine decreased from $1.26 \pm 0.29 \mathrm{mg} / \mathrm{dl}$ to $1.06 \pm 0.21 \mathrm{mg} / \mathrm{dl}$ (comparing to initial level). In spironolactone group serum creatinine also decreased from $1.26 \pm 0.31 \mathrm{mg} / \mathrm{dl}$ at the end of the study. In both groups it was statistically significant $(\mathrm{P}<0.01)$ but the differences between these two groups were not significant $(\mathrm{P}=0.81)$.

Estimated Marginal Means of MEASURE_1

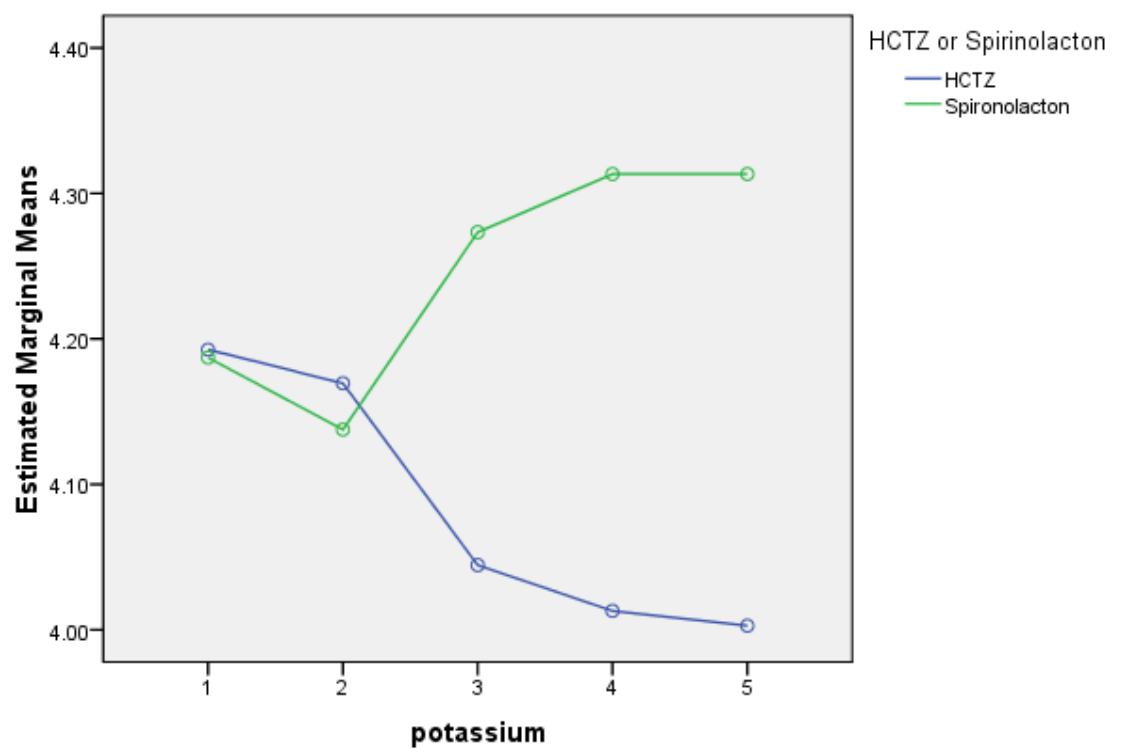

Fig.1 Serum potassium 
Int.J.Curr.Res.Aca.Rev.2016; 4(4): 225-233

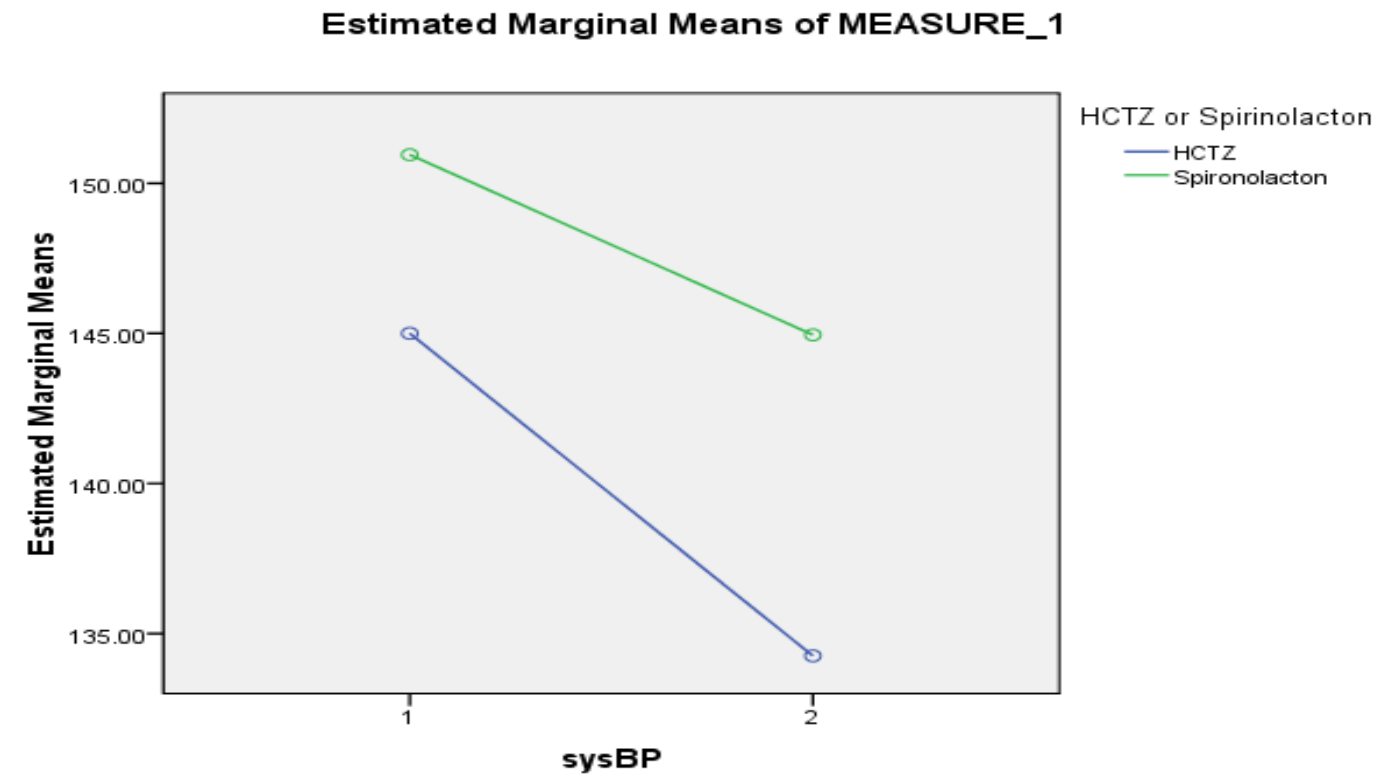

Fig.2 Systolic BP

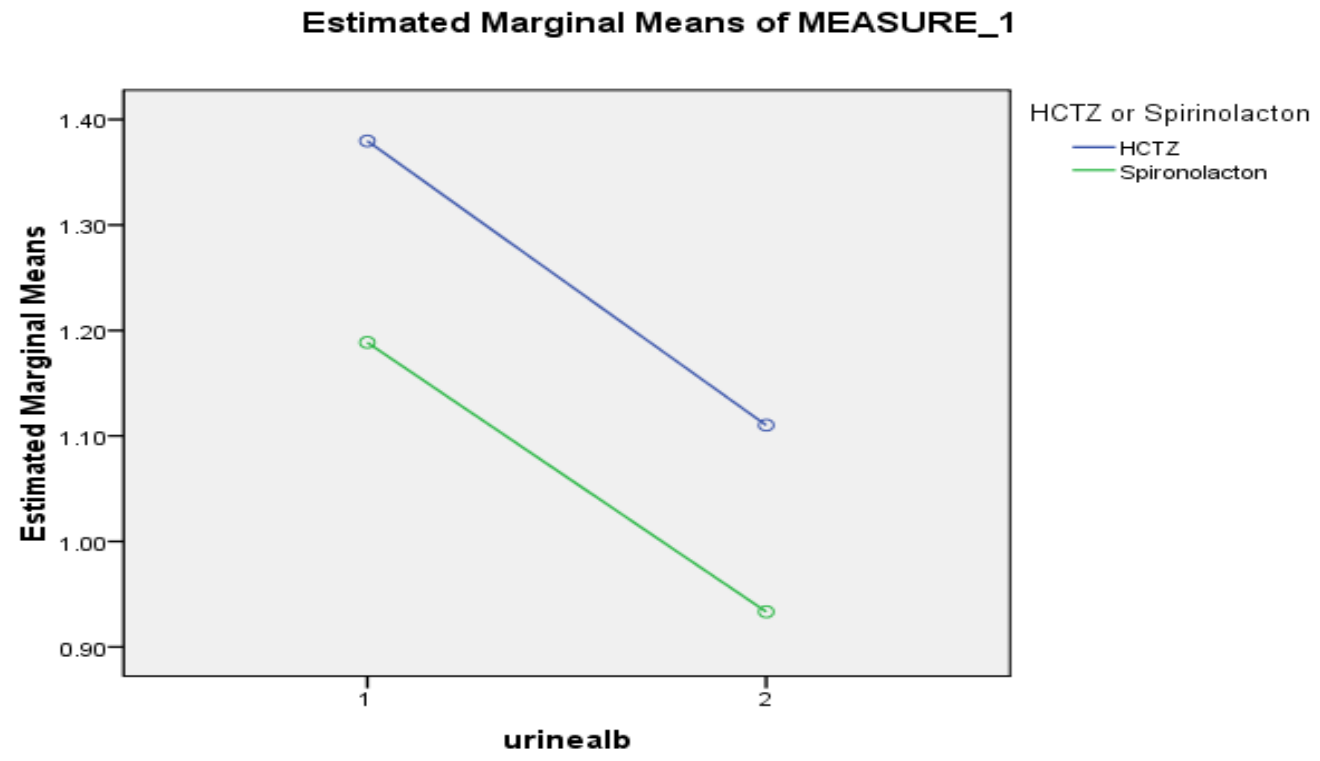

Fig.3 Urine albumin 
Int.J.Curr.Res.Aca.Rev.2016; 4(4): 225-233

Table.1 Results before and after intervention

\begin{tabular}{lccc}
\hline & $\begin{array}{c}\text { Beginning of } \\
\text { the study }\end{array}$ & $\begin{array}{c}\text { End of the } \\
\text { study }\end{array}$ & P value \\
\hline 24 hr urine albumin (mg/24hr) (HCTZ) & $\begin{array}{l}1342 \pm 927 \\
1093 \pm 814\end{array}$ & \\
24 hr urine albumin (mg/24hr) & $1183 \pm 916$ & $955 \pm 786$ & 0.33 \\
(spironolactone) & & & \\
Serum albumin (gr/dl) (HCTZ) & $3.43 \pm 0.38$ & $3.55 \pm 0.35$ & 0.79 \\
Serum albumin (gr/dl) (spironolactone) & $3.39 \pm 0.43$ & $3.53 \pm 0.35$ & \\
Systolic blood pressure (mmHg) (HCTZ) & $145 \pm 15$ & $135 \pm 16$ & \\
Systolic blood pressure (mmHg) & $151 \pm 12$ & $145 \pm 16$ & 0.02 \\
(spironolactone) & & & \\
Diastolic blood pressure (mmHg) (HCTZ) & $86 \pm 8$ & $77 \pm 6$ & \\
Diastolic blood pressure (mmHg) & $87 \pm 8$ & $78 \pm 7$ & 0.33 \\
(spironolactone) & & & \\
Serum potassium (mEq/L) (HCTZ) & $4.24 \pm 0.33$ & $4 \pm 0.19$ & 0.01 \\
Serum potassium (mEq/L) (spironolactone) & $4.19 \pm 0.33$ & $4.31 \pm 0.32$ & \\
Serum creatinin (mg/dl) (HCTZ) & $1.26 \pm 0.28$ & $1.06 \pm 0.21$ & 0.81 \\
Serum creatinin (mg/dl) (spironolactone) & $1.26 \pm 0.31$ & $1.05 \pm 0.21$ & \\
Serum urea (mg/dl) (HCTZ) & $33.13 \pm 9.67$ & $38.44 \pm 9.03$ & 0.79 \\
Serum urea (mg/dl) (spironolactone) & $33.19 \pm 10.55$ & $38.27 \pm 9.82$ & \\
GFR(cc/min) (HCTZ) & $63.8 \pm 8.76$ & $77.35 \pm 10.26$ & 0.61 \\
GFR(cc/min) (spironolactone) & $67.33 \pm 10.06$ & $75.13 \pm 10.36$ & \\
HbA1C (\%) (HCTZ) & $6.52 \pm 0.84$ & $6.19 \pm 0.7$ & 0.96 \\
HbA1C (spironolactone) & $6.3 \pm 0.67$ & $6.4 \pm 0.79$ & \\
\hline
\end{tabular}

Diabetic nephropathy is considered as one of most important leading causes of the ESRD all over the world. RAAS system plays an important role in kidney damage in diabetic patients. Proteinuria reduction with antihypertensive drugs in diabetic patients has shown that decreases the risk of ESRD development significantly $(5,7)$. ACEIs and ARBs both are choice antihypertensive drugs in hypertensive diabetic patients with diabetic nephropathy. When blood pressure level could not appropriately be controlled by each of the above mentioned drugs, another drug must be added to regimen $(5,7,11)$.

Every drug that potentially targets RAAS, reduces the progression of diabetic nephropathy theoretically because this system has the most important role in the pathogenesis of diabetic nephropathy $(5,7,11)$. Recent studies demonstrated that, the antiproteinuric effect of spironolactone is not completely related to its antihypertensive effect $(12,13,14)$.

Because of the known positive effects of HCTZ and spironolactone on reducing of proteinuria, the aim of this study was to compare the antiproteinuric effects of HCTZ and Spironolactone in this patients to choose the best second line treatment beside the ACEIs in these patients $(5,7,8-14)$.

In this study the range of albuminuria was significantly decreased in both groups (HCTZ and spironolactone), but there were no statistically significant differences $(\mathrm{P}$ value $=0.33$ ). The serum albumin was increased in both groups, but there were no 
significant differences between two groups either $(\mathrm{P}=0.79)$. SBP was more decreased in HCTZ group $(\mathrm{P}=0.02)$. Furthermore changes in diastolic blood pressure (DBP) were similar in both groups $(\mathrm{P}=0.33)$. Although, there was not any relationship between changes in systolic blood pressure (SBP) and improving of proteinuria $(\mathrm{P}=$ 0.71). This finding indicates that, the antiproteinuric effects of HCTZ are not absolutely because of its antihypertensive effects.

Similar findings were obtained in previous studies. In Bakris et al study, the antiprotienuric and antihypertensive effects of ACEIs and HCTZ combination was compared with ACEIs and amlodipine combination. They included 332 hypertensive patients with type $2 \mathrm{DM}$ and albuminuria. Patients were randomly divided into two subgroups. Benazepril and Amlodipine were prescribed to first group and second group received Benazepril and HCTZ combination. The study showed that, although patients who received Amlodipine and Benazepril had lower blood pressures, the albuminuria was more decreased in patients treated with HCTZ and Benazepril combination. This also may be due to an unknown anti-proteinuric effect of HCTZ (other than anti-hypertensive effect) (11).

Multiple studies have approved the positive antiproteinuric effects of HCTZ. Vogt and his colleagues in 2008 evaluated the effects of diets sodium content and HCTZ on the antiproteinuric effects of Losartan. In this study 34 non diabetic patients with proteinuria were included. The study showed that, low sodium diet and HCTZ both increase the antihypertensive and antiproteinuric effects of Losartan. Furthermore the study revealed that the combination of HCTZ, Losartan and low sodium diet is more effective than each one alone, but they did not use another drug to compare with HCTZ (9). Seven non diabetic patients with proteinuria that were treated with ACEIs for at least 3 months were included in a study by Buter and his colleagues. The study was consisting 3 steps. In the first step, patients were received low sodium diet (50 mmol per day) for 4 weeks. Then patients gave high sodium diet (200 mmol per day) for 4 weeks and finally patients were received high sodium diet in combination with HCTZ treatment. At the end of the each step, 24 hours urine samples were collected to evaluate the range of proteinuria. The study showed that, the antiproteinuric effect of ACEIs was reduced by high sodium diet, but improved by adding HCTZ. By the way they did not compare the effects of HCTZ with other drugs (10).

In another study, Velascoa et al compared antihypertensive and antiproteinuric effects of high dose Losartan and HCTZ with each other. In this study 90 hypertensive patients with type 2 diabetes were included. All antihypertensive drugs of patients were discontinued for 30 days. Then all patients received Losartan 50mg daily. Four weeks later, 22 patients had blood pressures lower than 130/85 $\mathrm{mmHg}$ and they continued with Losartan. The other 68 patients were randomly divided into two groups. First group received high dose Losartan $(100 \mathrm{mg}$ daily) in contrast; HCTZ (12.5 mg daily) was prescribed to the second group for 4 weeks. This study revealed that combination of HCTZ and Losartan has the similar effects on proteinuria as high dose Losartan alone but in our study only HCTZ was added to ACEIs and high dose Losartan was compared with HCTZ and Losartan combination (14).

In our study, serum potassium level increased significantly in some patients because of that 9 patients in spironolactone were excluded. In contrast, no potassium 
abnormalities were found among patients who were treated with HCTZ. This finding was similar to Chrysostomou et al study. In their research, the differences between combination therapy with ACEIs or ARBs and spironolactone were evaluated. The study revealed that the combination of Ramipril and spironolactone had the most positive effect on proteinuria. The study also revealed that, the combination of ACEIs, ARBs and spironolactone increases the risk of hyperkalemia (7). These findings emphasized that, HCTZ is better choice in combination therapy with ACEIs in comparison with spironolactone. These results were similar to ours.

In another study by Momeni et al, they found that the antiproteinuric effect of Spironolactone alone and HCTZ plus Spironolactone combination was more significant than HCTZ alone; against our study's results (5).

In some recent studies, impaired glucose tolerance was noticed among patients who were treated with HCTZ, but we did not find any glucose intolerances in our study (15, 16).

Our study revealed that, both spironolactone and HCTZ have similar antiproteinuric effects, but because HCTZ does not increase serum potassium level, it seems to be a better choice in combination therapy with ACEIs.

Limitations: Using of greater sample size mad longer follow up period may alter some of the results.

\section{Conclusion}

Our study showed that antiproteinuric effects of HCTZ and spironolactone were similar in hypertensive diabetic patients who were receiving ACEIs. Hyperkalemia was a serious side effect of spironolactone in combination with ACEIs and ARBs so close electrolyte control is needed. for this reason it seems that combination of HCTZ with these drugs is preferred.

\section{References}

1. Ozougwu, J.C, Obimba, K.C, Belonwu, C.D, Unakalamba, C.B. The pathogenesis and pathophysiology of type 1 and type 2 diabetes mellitus. Journal of physiology and pathophysiology. 2013; 4(4), 46-57.

2. Shaw J.E, Sicree P.Z, Zimmet P.Z Global estimates of the prevalence of diabetes for 2010 and 2030. Diabetes Res Clin Pract.2010;87(1):4-14.

3. Concannon P, Rich S, Nepom G. Genetics of type 1A diabetes. N Engl J Med.2009; 360,1646-1654.

4. Gerstein HC, Miller ME, Byington RP, et al. Effects of intensive glucose lowering in type 2 diabetes. $N$ Engl $J$ Med.2012; 358(24),2545-2559.

5. Momeni A, Behradmanesh MS, Kheiri S, Karami Horestani M. Evaluation of spironolactone plus hydrochlorothiazide in reducing proteinuria in type 2 diabetic nephropathy. Journal of the Renin-Angiotensin-Aldosterone System: 2013;1-6.

6. Prasad P, Tiwari AK, Kumar KM, et al. Chronic renal insufficiency among Asian Indians with type 2 diabetes: I. Role of RAAS gene polymorphisms. BMC MedGenet, 2006, 7:42.

7. Chrysostomou A, Pedagogos E, MacGregor L. Double-Blind, PlaceboControlled Study on the Effect of the Aldosterone Receptor Antagonist Spironolactone in Patients Who Have Persistent Proteinuria and Are on LongTerm Angiotensin-Converting Enzyme Inhibitor Therapy, with or without an 
Angiotensin II Receptor Blocker. Clin J Am Soc Nephrol.2006;1: 256-262.

8. Abe M, Okada K, Maruyama T, Matsumoto K. Antiproteinuric and blood pressure-lowering effects of a fixed-dose combination of losartan and hydrochlorothiazide in hypertensive patients with stage 3 chronic kidney disease. Pharmacotherapy. 2009;29 (9):1061-1072.

9. Vogt L, Waanders F, Boomsma F. Effects of Dietary Sodium and Hydrochlorothiazide on the Antiproteinuric Efficacy of Losartan. $J$ Am Soc Nephrol.2008;19: 999-1007.

10. Buter H, Hemmelder MH, Navis G. The blunting of the antiproteinuric efficacy of ACE inhibition by high sodium intake can be restored by hydrochlorothiazide. Nephrol Dial Transplant.1998;13: 1682-1685

11. Bakris GL, Toto RD, McCullough PA. Effects of different ACE inhibitor combinations on albuminuria: results of the GUARD study. Kidney International.2008;73: 1303-1309.

12. Rossing K, Schjoedt KJ, Smidt UM,et al.. Beneficial Effects of Adding Spironolactone to Recommended Antihypertensive Treatment in Diabetic Nephropathy. Diabetes Care.2005; 28:2106-2112.

13. Schjoedt KJ, Rossing K, Juhl TR. Beneficial impact of spironolactone in diabetic nephropathy. Kidney International.2005; 68:2829-2836.

14. Velascoa PL, Toralb FP, Esmatjes JE. Losartan titration versus diuretic combination in type 2 diabetic patients. Journal of Hypertension.2002; 20(4):715-719.

15. Sears AJ, Woods SH, Watts MM, et al. A double-blind, placebo controlled, crossover trial comparing the effect of amloride and hydrochlorthiazide on glucose tolerance in patients with essential hypertension. Hypertension. 2012; 59: 934.

16. Elliot WJ. Effects of potassium-sparing versus thiazide diuretics on glucose tolerance: New data on an old topic. Hypertension.2012;59: 911.

17. Dai HY, Zheng M, Tang RN, et al. Effects of angiotensin receptor blocker on phenotypic alterations of podocytes in early diabetic nephropathy. Am J Med Sci.2011; 341:207-14.

18. Appel GB. Preventing or slowing the progression of diabetic nephropathy. BUMC Proceedings, 1992; 12(1): 3-6.

19. Molitch ME, DeFronzo RA, Franz MJ, et al. Nephropathy in diabetes. Diabetes Care, 2004;27 Suppl 1:S79-83.

20. Bach LA, Gilbert RE, Cooper ME, Tsalamandris C, Jerums G. Prediction of persistent microalbuminuria in patients with diabetes mellitus. $J$ Diabetes Complications, 1993;7(2):6772.

21. Naidoo DP. The link between microalbuminuria, endothelial dysfunction and cardiovascular disease in diabetes. Cardiovasc $\mathrm{J} S$ Afr. 2002;13(4):194-9.

\section{How to cite this article:}

Hamid Noshad, Taher Manzary and Amir Teimouri-Dereshki. 2016. Effect of hydrochlorothiazide and spironolactone on reducing proteinuria among patients with diabetic nephropathy treated by angiotensin converting enzyme inhibitors. Int.J.Curr.Res.Aca.Rev.4(4): 225-233. doi: http://dx.doi.org/10.20546/ijcrar.2016.404.028 